\title{
Change of the SOL Power Width with the Toroidal B-Field Direction in ASDEX Upgrade
}

\author{
M.Faitsch ${ }^{1}$, B.Sieglin ${ }^{1}$, T.Eich ${ }^{1}$, H.J.Sun ${ }^{1}$, A.Herrmann ${ }^{1}$ and \\ the ASDEX Upgrade Team \\ ${ }^{1}$ Max-Planck-Institute for Plasma Physics, Boltzmannstr. 2, D-85748 Garching, \\ Germany \\ E-mail: Michael.Faitsch@ipp.mpg.de
}

\begin{abstract}
The change of the scrape-off layer power width in dependence on the toroidal magnetic field direction is investigated in L-mode discharges in upper single null (USN) configuration in ASDEX Upgrade. The heat flux onto the outer and inner divertor plates is measured using a fast $2 \mathrm{D}$ infrared camera. The heat flux distribution is described by an exponential power fall-off length $\lambda_{q}$ and a diffusive broadening in the divertor region $S$. In this paper the parameters, $S$ and $\lambda_{q}$, for the inner and outer divertor target are compared for both toroidal magnetic field directions. For the divertor broadening $S$ no dependence on the toroidal magnetic field direction is observed. The comparison between the near scrape-off layer electron temperature fall-off length $\lambda_{T_{e}}$ and the power fall-off length $\lambda_{q}$ are in agreement with the 2-point model. It is concluded that electron conduction is the main contribution for the scrape-off layer parallel transport in these discharges. The ratio between inner, $\lambda_{q}^{\text {inner }}$, and outer, $\lambda_{q}^{\text {outer }}$, power fall-off length is dependent on the toroidal magnetic field direction. The numerical values are $\lambda_{q}^{\text {inner }} / \lambda_{q}^{\text {outer }}=0.44$ for favourable $\mathbf{B} \times \nabla B$ ion drift direction and $\lambda_{q}^{\text {inner }} / \lambda_{q}^{\text {outer }}=0.85$ for non-favourable drift direction. The different ratios are explained by vertical drifts, which are dependent on the toroidal magnetic field direction.
\end{abstract}

Submitted to: Plasma Phys. Control. Fusion

\section{Introduction}

One major challenge for future fusion devices, such as ITER and DEMO, is power exhaust [1, 2]. So far most scrape-off layer studies were reported for the outer divertor and the ion $\nabla B$ drift towards the active $\mathrm{X}$-point, often called normal or favourable field direction. With this magnetic field direction the $\mathrm{L}-\mathrm{H}$ power threshold is lower $[3,4,5,6]$ and therefore this is the foreseen drift direction in future fusion devices. It is reported $[7,8]$ that more power is deposited onto the outer divertor target making it the critical area in the divertor in such scenarios. However, for the understanding of the scrape-off layer transport forming the power fall-off length $\lambda_{q}$ and the divertor broadening $S$, measurements on both divertor targets as well as the change in the 
toroidal magnetic field direction are desirable. In [9] studies with both magnetic field directions are reported for JET. It highlights the importance of drifts onto the scrape-off layer transport, focusing mainly on the asymmetry between the energy distributed onto the inner and outer divertor in both L- and H-mode.

\section{Experimental setup}

ASDEX Upgrade is a medium-sized divertor tokamak [10]. ASDEX Upgrade can perform discharges in lower single null (LSN), in double null (DN) and upper single null (USN). The upper divertor is an open divertor with tungsten coated fine grain graphite tiles. The geometry is illustrated in figure 1. The upper divertor tiles are not toroidally tilted. This makes it possible to change the toroidal magnetic field direction without having to change the plasma current direction to keep the helicity. Discharges with both toroidal magnetic field directions are presented in this paper to study the effect of magnetic drifts onto the power fall-off length $\lambda_{q}$ and the divertor broadening $S$.

To be able to compare different divertor geometries and devices the measured values on the divertor targets are mapped to the outer mid-plane by normalizing it using the effective flux expansion $f_{x}$ [11]. The data measured on the inner divertor target is mapped to the outer mid-plane as well. The flux expansion in real space from the outer mid-plane to the inner mid-plane (IMP) is around $f_{x, I M P}=1.5$. This leads to, on average, a higher flux expansion for the inner divertor compared to the outer divertor in the configuration used for the presented discharges.

The infrared (IR) system measures at a wavelength of $4.7 \mu \mathrm{m}$. The target resolution for the inner target is $1.6 \mathrm{~mm} /$ pixel and for the outer target $2.2 \mathrm{~mm} /$ pixel. The frame rate is between 350 and $780 \mathrm{~Hz}$. The heat flux is calculated using the THEODOR code [8]. The studies are performed with a plasma current of $I_{p}=1 \mathrm{MA}$ and a toroidal magnetic field strength of $\left|B_{t}\right|=2.5 \mathrm{~T}$. The line integrated core electron density $n_{e, \text { core }}$ is varied between 2.0-6.0 $\cdot 10^{19} \mathrm{~m}^{-2}$, measured with the DCN interferometer channel H-1 [12]. The line of sight of this channel is shown in figure 1. The auxiliary heating power is around $0.75 \mathrm{MW}$ for positive toroidal magnetic field and around 1.3 MW for negative toroidal magnetic field using central ECRH. The higher heating power for negative toroidal magnetic field partially compensates the higher energy losses in the non-favourable magnetic field direction, thus having roughly the same magnetohydrodynamic stored energy, $W_{M H D}$ from CLISTE[13], for both, favourable and non-favourable drift direction. Time traces for two discharges with different toroidal magnetic field direction are shown in figure 2. In USN H-mode the inner divertor is in most cases detached and therefore no comparison between the measurements on both divertor targets is possible. The studies shown in this paper are performed in L-mode were both divertor targets are attached and the power deposition can be compared. 


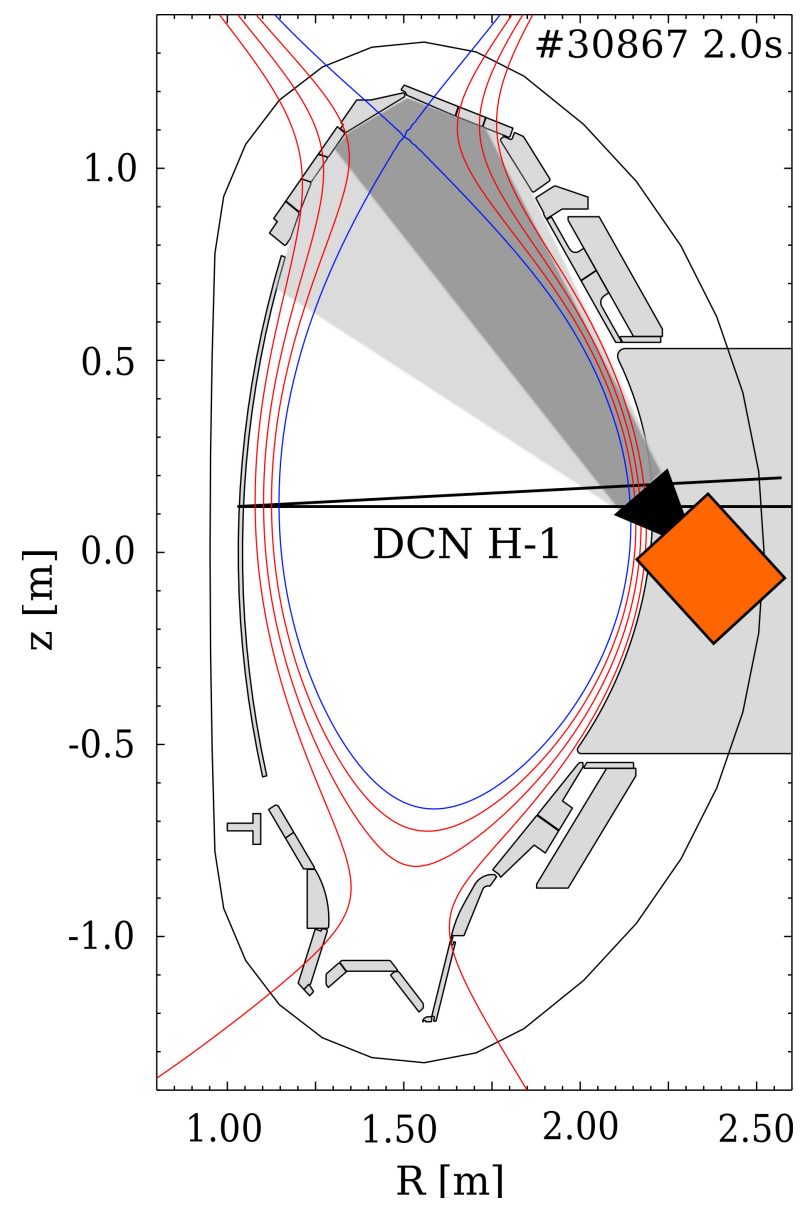

Figure 1. Poloidal cross-section of ASDEX Upgrade showing also the IR view as well as the utilized DCN channel H-1. The brighter grey area is approximately the full view poloidal volume, the darker grey the measurement view with the smaller frame size for an increased time resolution.

\section{Experimental results}

In this section the experimental results for the parameters $\lambda_{i n t}, S$ and $\lambda_{q}$ for the Lmode plasmas are discussed. In some discharges the heat flux or the signal to noise level is too low for the measured profiles on the inner divertor target. Further has to be stated that no energy balance is possible for the discharges presented. This is due to the generally low inclination angles of the field lines on the upper divertor in the order of $2^{\circ}$ in combination with toroidal flat divertor tiles causing leading edges. The heat flux distribution is hence not toroidally symmetric and the local power flux cannot be toroidally integrated to estimate a power balance. Calculating the heat flux onto different toroidal positions in order of getting a toroidally averaged heat flux is not possible due to too low signal to noise ratio in toroidal positions with lower but still relevant heat flux. 

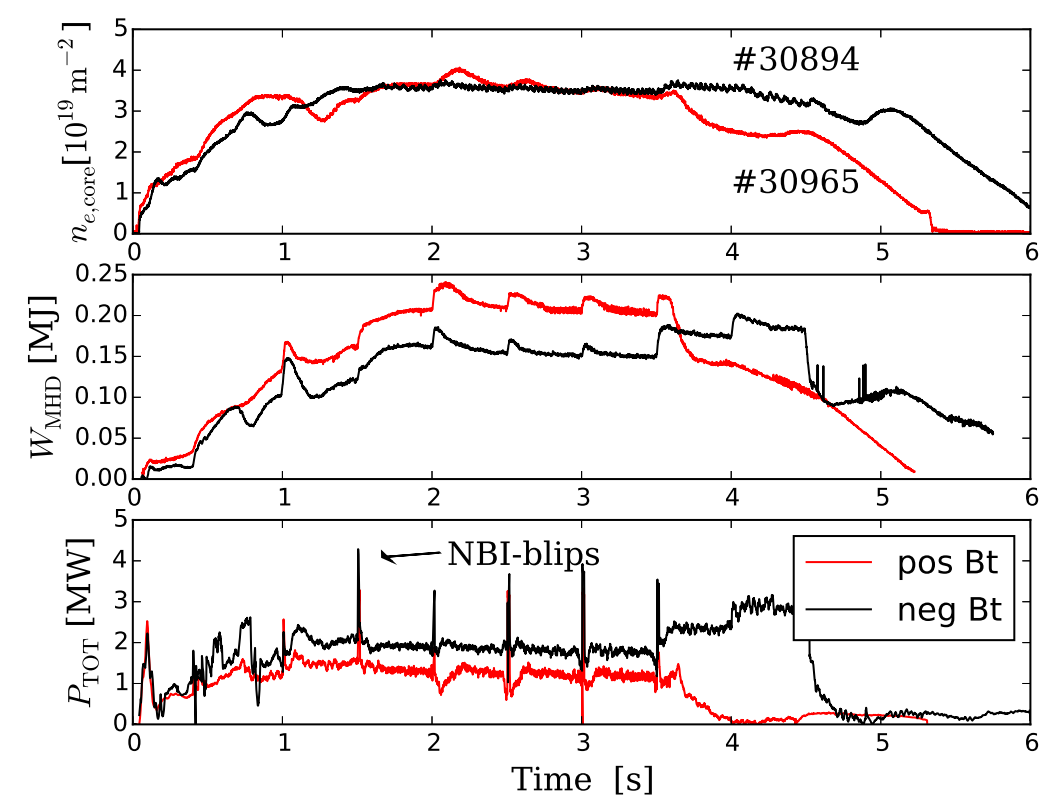

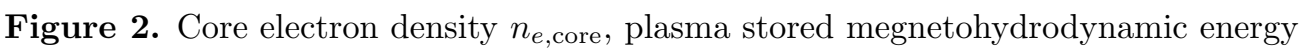
$W_{M H D}$ and total heating power $P_{T O T}$ for discharges \#30894 (negative toroidal field, unfavourable drift direction) and \#30965 (positive toroidal field, favourable drift direction).

\subsection{Integral power decay width}

The integral power decay width $\lambda_{\text {int }}$ is an important quantity for the divertor power load:

$$
\lambda_{\text {int }}=\frac{\int\left(q(s)-q_{B G}\right) d s}{q_{\max }-q_{B G}} \cdot f_{x}^{-1}[\mathrm{~mm}]
$$

with $q(s)$ the heat flux density along the target coordinate $s, q_{B G}$ the constant background heat flux and $q_{\max }$ the peak heat flux on target.

For the heat flux profile on the divertor targets a diffusive model is presented in [11] which is compared to data in figure 3.

$$
q(s)=\frac{q_{0}}{2} \exp \left(\left(\frac{S}{2 \lambda_{q}}\right)^{2}-\frac{s}{\lambda_{q} f_{x}}\right) \cdot \operatorname{erfc}\left(\frac{S}{2 \lambda_{q}}-\frac{s}{S f_{x}}\right)+q_{B G}\left[\frac{\mathrm{MW}}{\mathrm{m}^{2}}\right]
$$

For this model a simple estimation of the integral power decay width is given by Makowski [14]:

$$
\lambda_{\text {int }} \approx \lambda_{q}+1.64 \cdot S[\mathrm{~mm}]
$$

This relation is used as a qualifier for both the data as well as the fit quality. Both the integral power decay width and the fitting parameters are shown in figure 4 for discharge \#30965 at 1.97 s. This comparison is shown in figure 5 for the whole data base used for this paper, for both the inner and outer divertor target. Here it shall be noted that the values for $\lambda_{\text {int }}$ for the inner and outer target are in the same range with slightly 


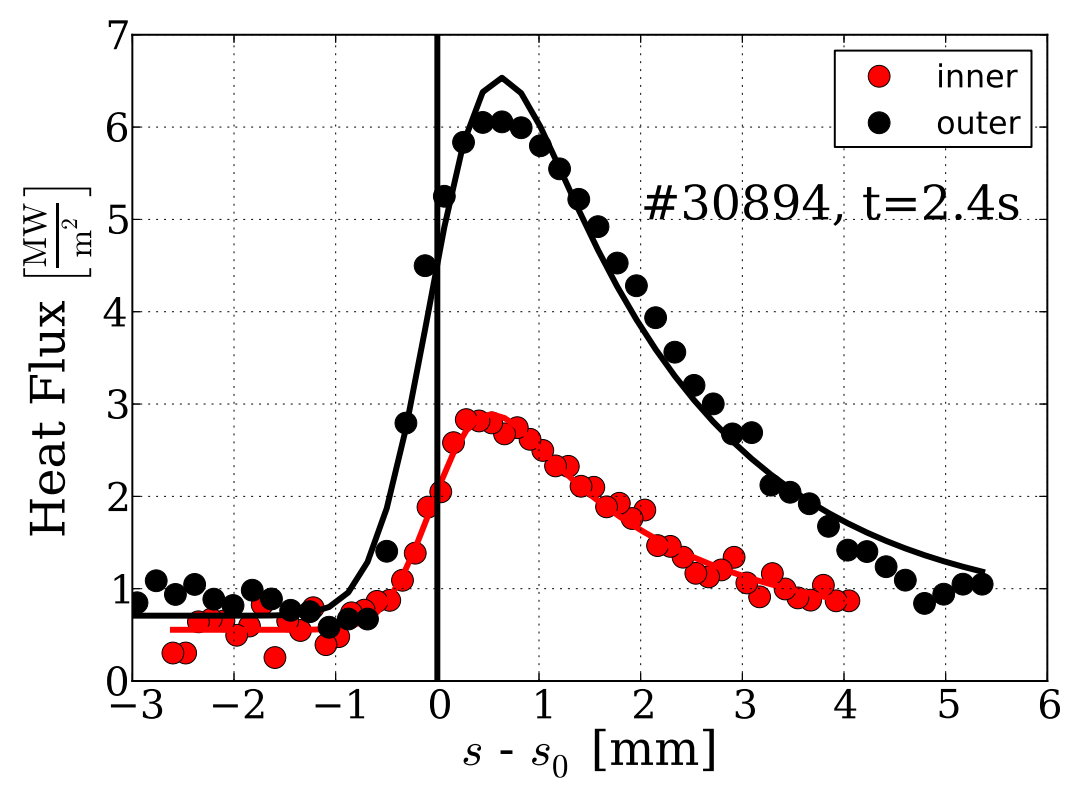

Figure 3. Heat flux profiles on both the inner and outer divertor target for discharge \#30894 at 2.4 s. In this discharge the toroidal magnetic field is negative, unfavourable drift direction.

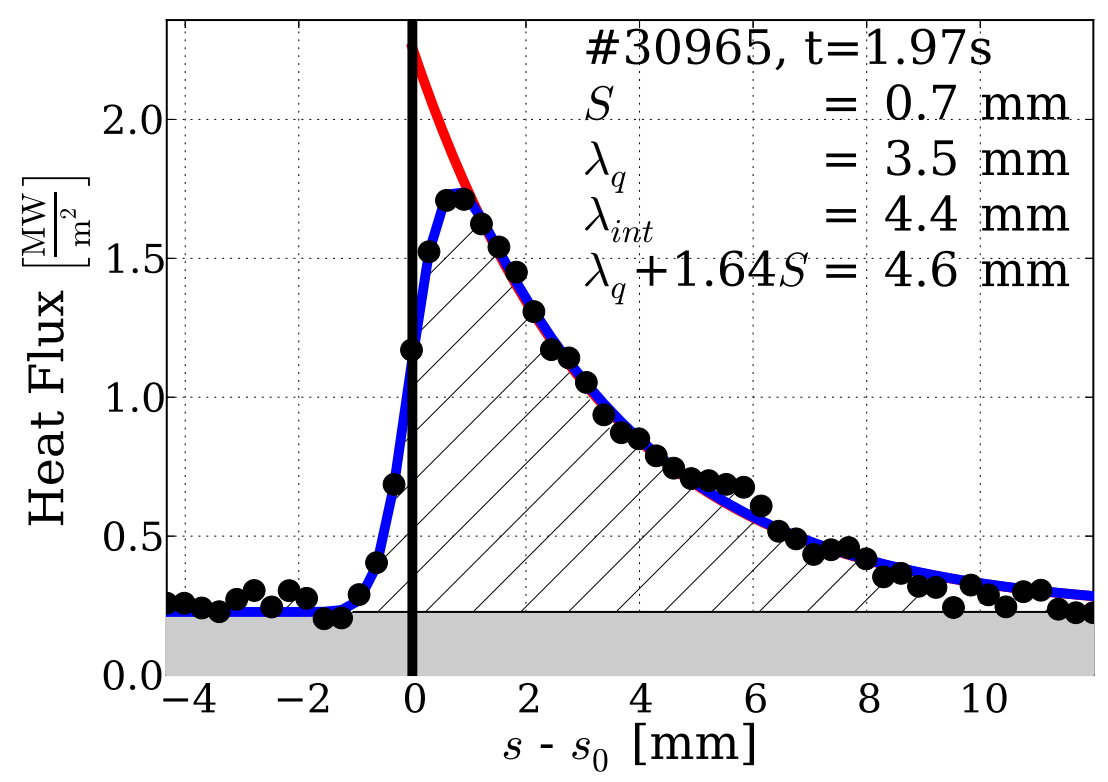

Figure 4. Integral (black stripes), model (blue line) and exponential decay (red line) for the outer divertor target for discharge \#30965 at 1.97 s. The grey area is the calculated background.

lower values for the inner target. A linear regression shows, that the values obtained using equation (3) are $3 \%$ larger than the values for $\lambda_{\text {int }}$ taken from equation (1). This 


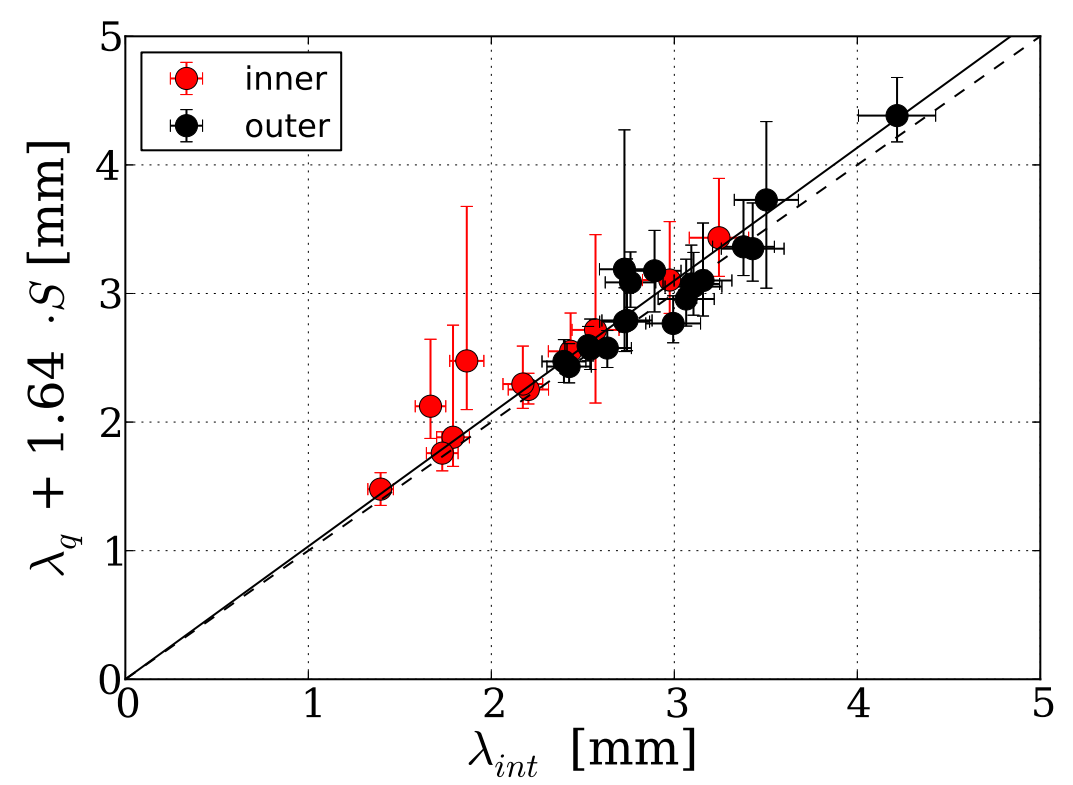

Figure 5. Comparison of the integral power decay (integration along the target coordinate, equation (1)) and the fitted parameters $\lambda_{q}, S$, equation (3), both mapped to the outer mid-plane. The dashed line is the identity, the solid line a linear regression with a slope of 1.03 .

deviation is discussed in the appendix.

\subsection{Divertor broadening $S$}

The measured values for the divertor broadening $S$ range between $0.4-1.0 \mathrm{~mm}$ with most values between $0.6-0.8 \mathrm{~mm}$ as shown in figure $6(\mathrm{a})$. No dependence on the core electron density is observed for this data base.

In figure $6(\mathrm{~b})$ the ratio between the divertor broadening $S$ for the inner and outer target is shown. For both toroidal magnetic field directions the values for the inner divertor are slightly higher. The ratios vary from 0.9 to 1.5 with a mean value of 1.2 for both field directions.

\subsection{Power fall-off length $\lambda_{q}$}

The values for $\lambda_{q}$ are shown in figure 7 . The variation for the outer target is between 1.5-3.5 $\mathrm{mm}$ and for the inner target between 0.6-2.4 mm. A difference between the inner and outer target is visible, namely smaller $\lambda_{q}^{i n n e r}$. As mentioned in the previous section the divertor broadening $S$ on the inner divertor is larger compared to the outer divertor. No dependence on the core electron density is observed.

The ratio between the inner and outer $\lambda_{q}$ is shown in figure 8 . No dependence on the core electron density for this ratio is observed. This ratio is dependent on the toroidal magnetic field direction. For negative toroidal magnetic field the inner and 


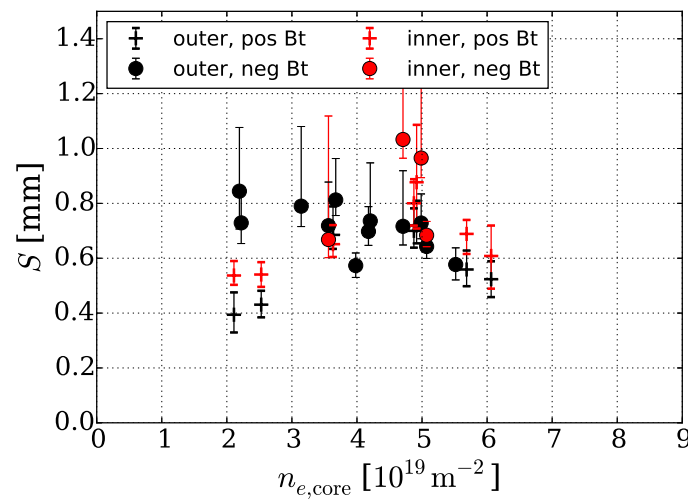

(a) Data from inner and outer target in dependence of the core electron density.

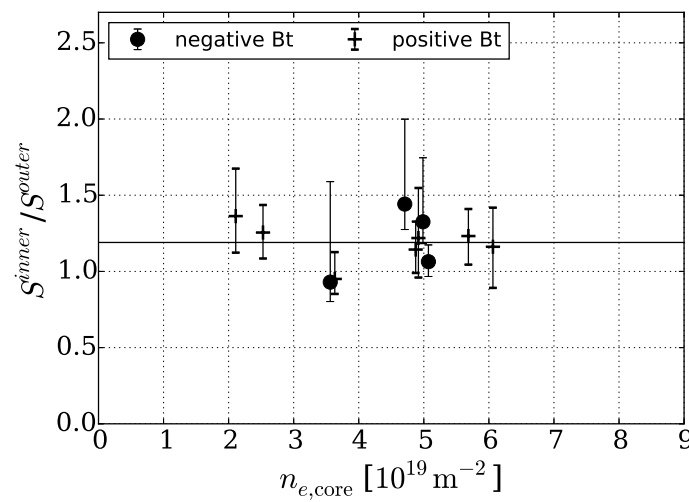

(b) Value for the inner target divided by the value for the outer target.

Figure 6. Divertor broadening $S$. No significant influence on the field direction nor the core electron density is detected for both the values for the inner and outer target as well as the ratio between inner and outer target.

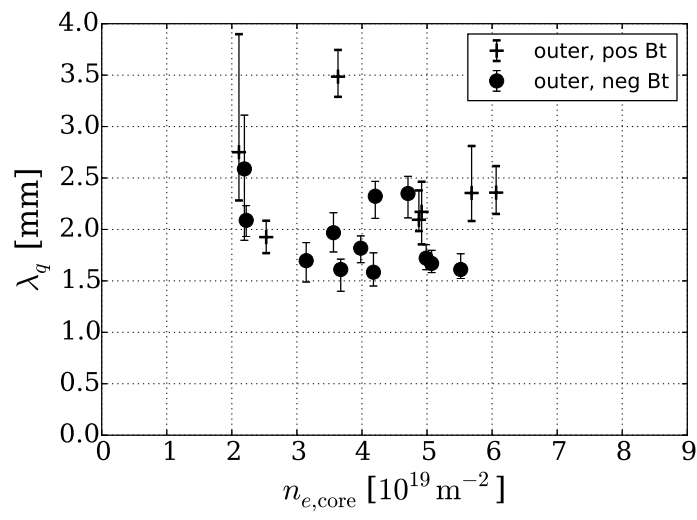

(a) outer target

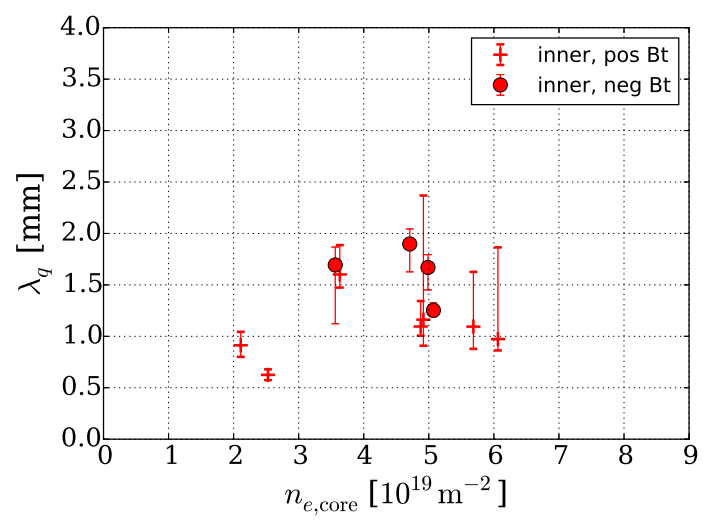

(b) inner target

Figure 7. Power fall-off length $\lambda_{q}$ on the inner and outer target in dependence of the core electron density.

outer power fall-off length $\lambda_{q}$ have a ratio of around 0.85. For positive toroidal magnetic field this ratio is around 0.44. For all discharges performed for this study, the power fall-off length $\lambda_{q}$ is larger on the outer target compared to the inner target. The divertor broadening $S$ shows the opposite behaviour with larger values for the inner target.

In figure 9 the values for this data base are compared to the scaling (equation (4)).

$$
\lambda_{q}^{\text {scaling }}=2 \cdot 0.73 \cdot B_{t}^{-0.78} \cdot q_{c y l}^{1.2} \cdot P_{S O L}^{0.1}[\mathrm{~mm}]
$$

with the cylindrical safety factor $q_{c y l}$ [11]. For the power crossing the separatrix $P_{S O L}=0.7 \cdot P_{T O T}$ is assumed, where $P_{T O T}$ is the total heating power. This scaling contains the H-mode scaling from [11] and an additional factor 2 for L-mode as mentioned in [15]. The L-mode scaling published in [15] predicts too large values. The 


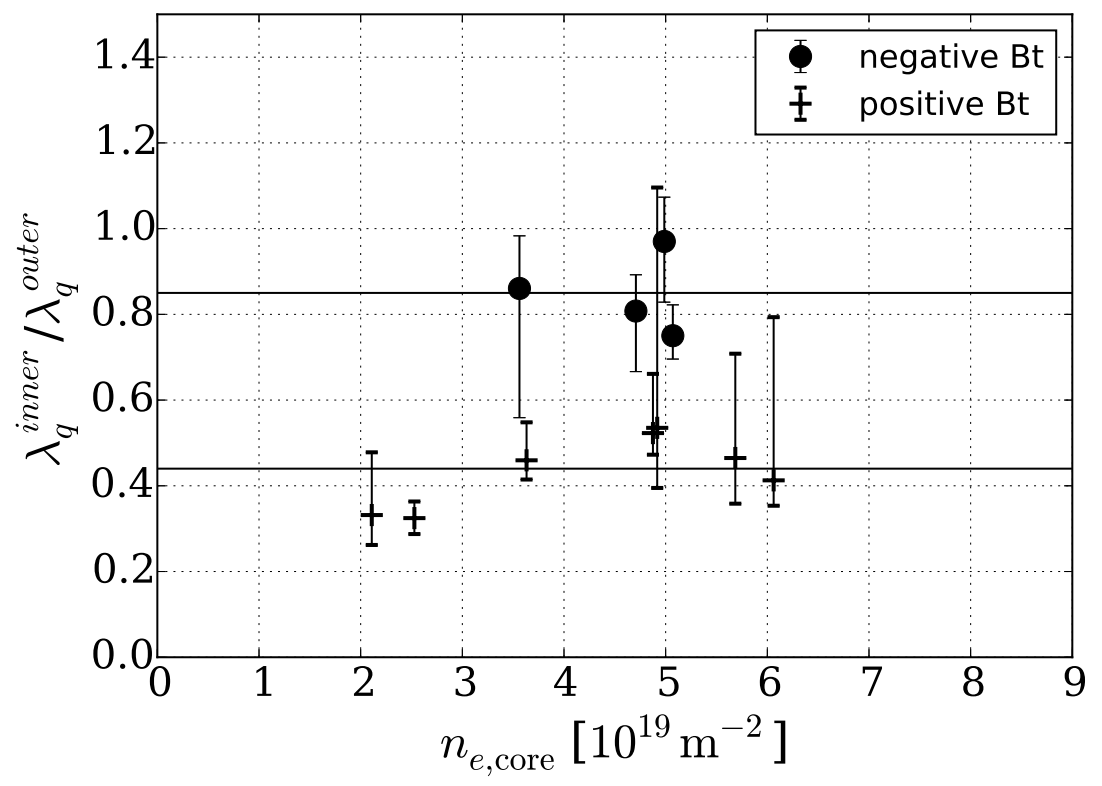

Figure 8. Power fall-off length $\lambda_{q}$ : value for the inner target divided by the value for the outer target in dependence of the core electron density.

main difference is a slightly weaker power dependence and $q_{c y l}$ as parameter instead of $q_{95}$ for the scaling compared to the L-mode scaling. The uncertainty of the H-mode

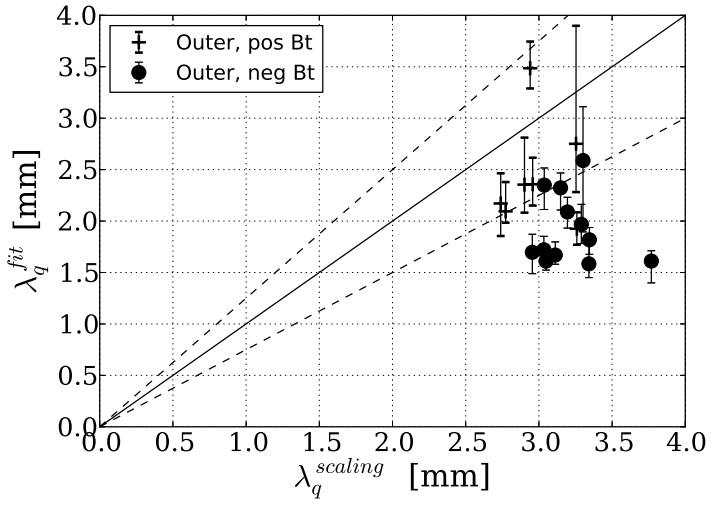

(a) outer target

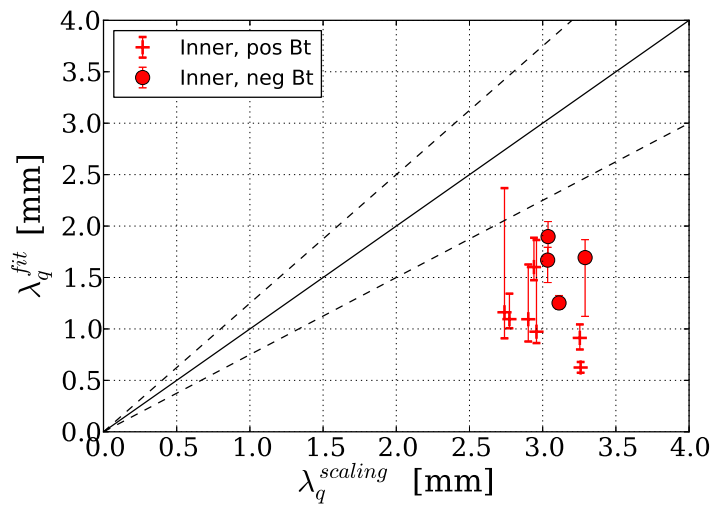

(b) inner target

Figure 9. Comparison between the divertor target power fall-off length $\lambda_{q}$ and the values obtained using equation (4).

scaling law prediction is $25 \%$ using the multi-machine data base [16]. This uncertainty is taken for the comparison between the fit results and the scaling results in figure 9 . It is represented with the dashed lines.

Only the data achieved with a positive toroidal magnetic field on the upper outer divertor are described by the scaling law. This is the normal field direction, which is used for 
the scaling laws published in $[11,15]$.

\section{Interpretation}

In the following section measurements of the near scrape-off layer temperature falloff length $\lambda_{T_{e}}$ using Thomson-Scattering are compared to the power fall-off length $\lambda_{q}$ measurements using IR thermography. Furthermore the influence of vertical particle drifts on the scrape-off layer power fall-off length $\lambda_{q}$ is discussed.

\subsection{Comparison of $\lambda_{q}$ with upstream measurements of $\lambda_{T_{e}}$}

The Thomson-Scattering measurement on ASDEX Upgrade [17] gives the opportunity to compare the power fall-off length $\lambda_{q}$ measured by IR thermography in the divertor with the exponential decay of the electron temperature $\lambda_{T_{e}}$ at the outer mid-plane in the near scrape-off layer. A typical profile is shown in figure 10. The separatrix position in ASDEX Upgrade H-mode is set to be $100 \mathrm{eV}$ as discussed in [18]. In L-mode the separatrix electron temperature is typically in the range of $50-60 \mathrm{eV}$ due to the lower parallel heat flux [18]. Assuming classical electron heat conduction, Spitzer-Härm

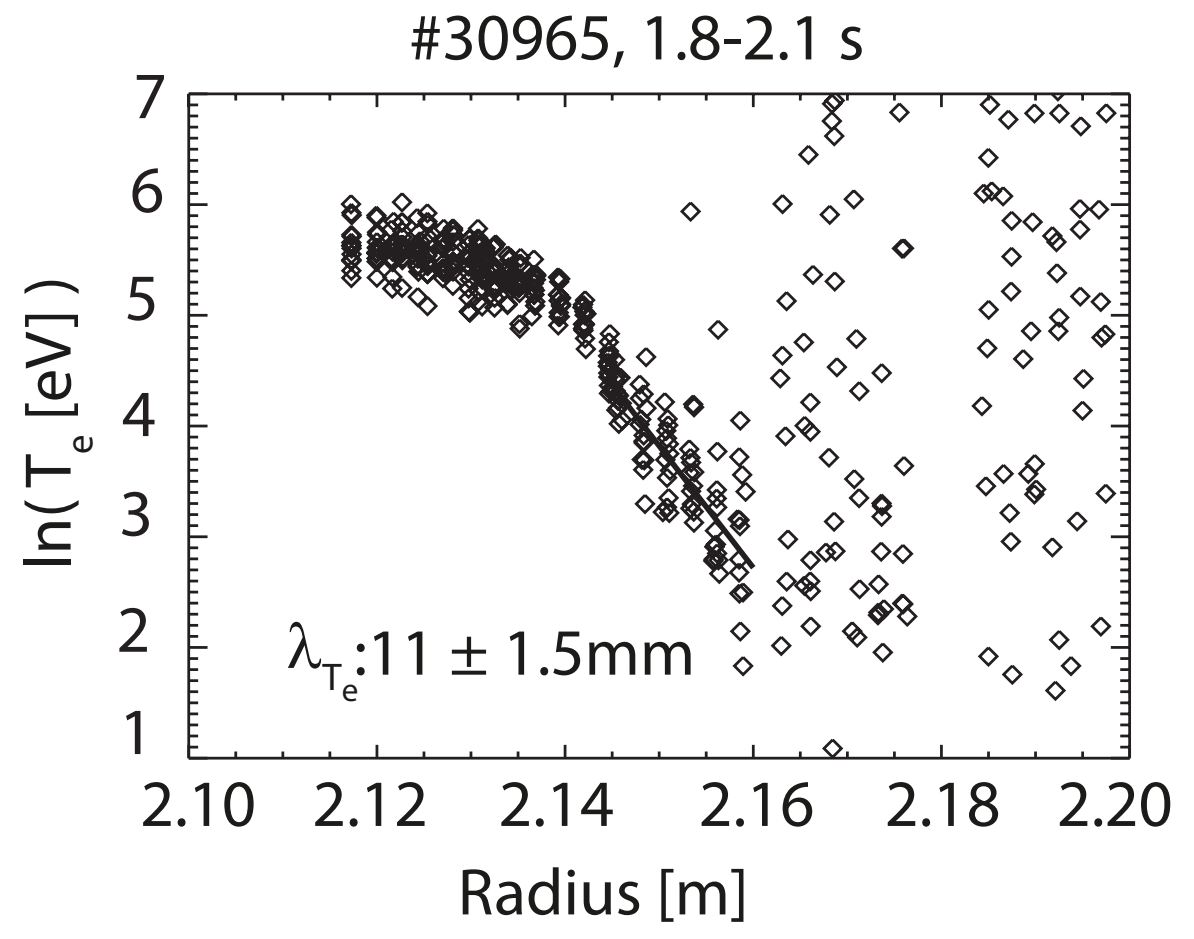

Figure 10. Electron temperature profile for \#30965 from $1.8 \mathrm{~s}$ to $2.1 \mathrm{~s}$. The near scrape-off layer electron temperature decay length is $11 \mathrm{~mm}$.

conductivity, for the parallel heat flux density $q_{||}$in the scrape-off layer leads to:

$$
q_{\|}=-\kappa_{0} T^{\frac{5}{2}} \frac{\mathrm{d} T}{\mathrm{~d} x}
$$


with $\mathrm{x}$ the coordinate along the field line [19]. Integrating along the field line under the assumption that the power enters the scrape-off layer predominantly at a localized area poloidally upstream the following relation for the upstream temperature $T_{u}$ is obtained [20]:

$$
T_{u}=\left(T_{t}^{\frac{7}{2}}+\frac{7 q_{||} L}{2 \kappa_{0}}\right)^{\frac{2}{7}}
$$

Neglecting the target electron temperature $T_{t}\left(T_{u}>>T_{t}\right)$ :

$$
T_{u} \approx\left(\frac{7 q_{\|} L}{2 \kappa_{0}}\right)^{\frac{2}{7}}
$$

leading to:

$$
\frac{\lambda_{T_{e}}}{\lambda_{q}}=\frac{7}{2}
$$

The ratio between the electron temperature width $\lambda_{T_{e}}$ measured by Thomson-Scattering and the power fall-off length $\lambda_{q}$ measured by IR thermography is shown in figure 11 .

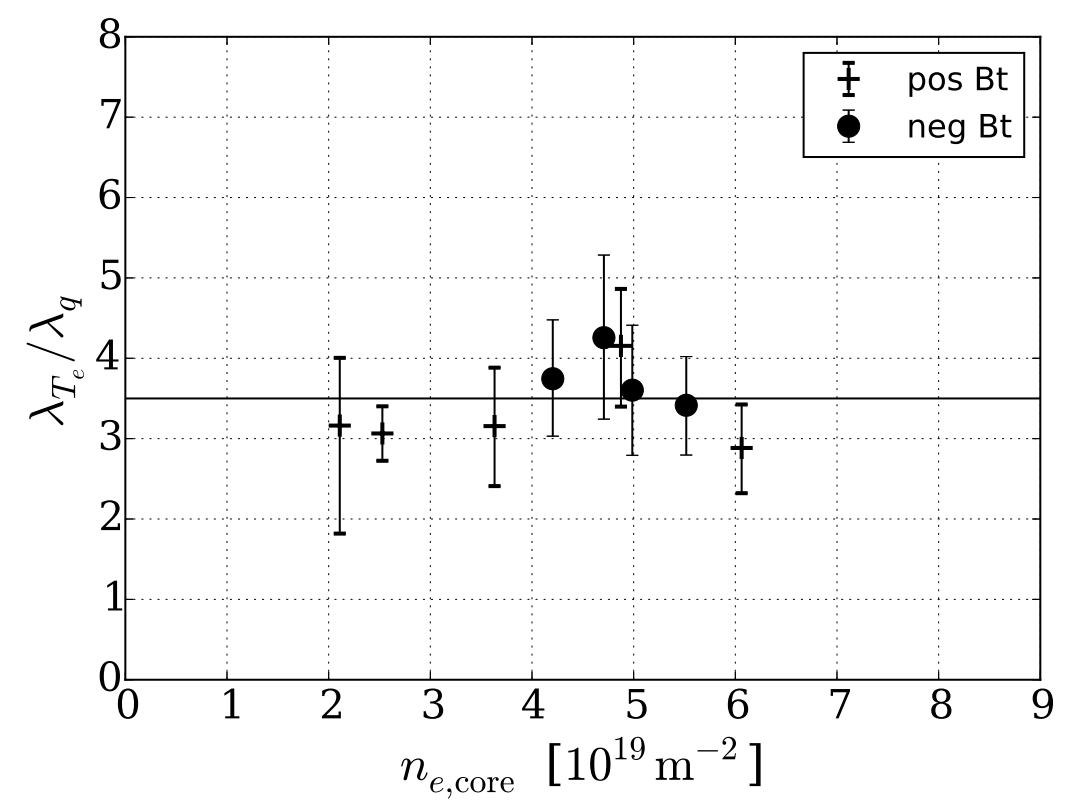

Figure 11. Ratio between $\lambda_{T_{e}}$, measured by Thomson scattering, and $\lambda_{q}$, measured by infrared thermography on the outer divertor target. The solid line represents a ratio of 3.5 , the ratio obtained using the 2-point model (equation (8)).

It is seen that the ratio between $\lambda_{T_{e}}$ and outer $\lambda_{q}$ matches the 2-point model within the uncertainty. Therefore the data base is in agreement with the assumption that the scrape-off layer transport from upstream to the outer divertor is dominated by electron heat conduction. However, with the on average smaller power fall-off length for the inner divertor, the measurements are not in agreement with a simple two-point model between the outer mid-plane and the inner divertor. The connection between the 
upstream electron temperature measured at the outer mid-plane and the inner power fall-off length is not clear. For the understanding of this we propose to investigate the power fall-off lengths $\lambda_{q}$ on the upper and lower outer divertor target in DN discharges to disentangle the drift effects from geometry effects possibly arising due to the about two times larger connection length to the inner target from the outer mid-plane.

\subsection{Influence of the plasma triangularity}

One way of interpreting the scrape-off layer width is the influence of particle drifts, e.g. the heuristic drift-based model by Goldston [21]. In this model vertical particle drifts, the grad B and curvature drift, carry particles across the separatrix onto open field lines. These drift flows are balanced by parallel flows connecting the top and the bottom of the plasma and by parallel flows leaving the plasma in the direction of the divertor. The scrape-off layer width is the radial displacement due to the vertical particle drifts. This is illustrated in figure 12. The blue filled arrows indicate the magnetic drift direction of the ions, the green open arrows the poloidal flow in the scrape-off layer. The purple lines are sketches of the poloidal cross-section of an ion orbit due to vertical drifts and the poloidal flow.

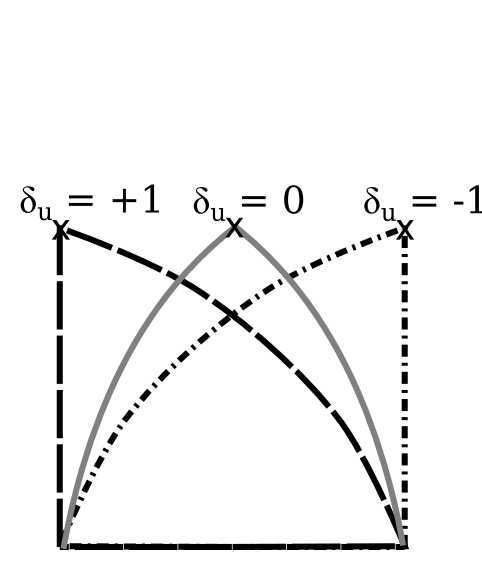

(a) Triangularity for the active $\mathrm{x}$-point

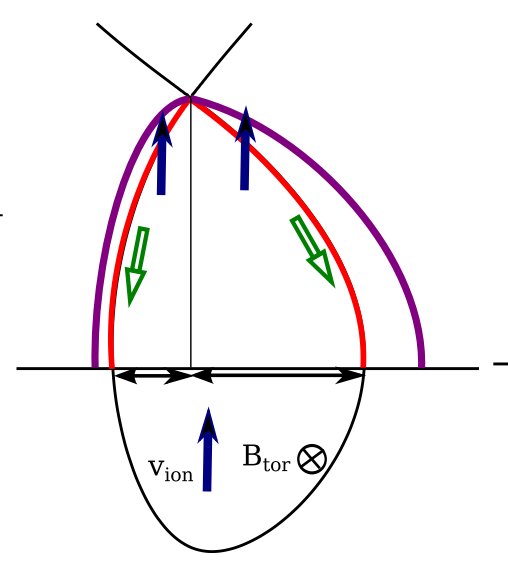

(b) normal field

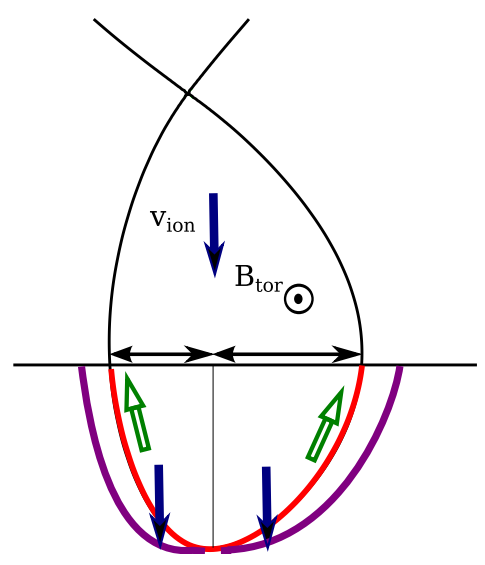

(c) reversed field

Figure 12. Influence of the magnetic drift direction onto the scrape-off layer width.

The triangularity is defined as in [22]:

$$
\begin{aligned}
& \delta_{\text {upper }}=\left(R_{\text {geo }}-R_{\text {upper }}\right) / a \\
& \delta_{\text {lower }}=\left(R_{\text {geo }}-R_{\text {lower }}\right) / a
\end{aligned}
$$

with the minor radius $a$, the geometrical major radius $R_{g e o}$ and the major radius of the lowest and highest point of the separatrix, $R_{\text {lower }}$ and $R_{\text {upper }}$. Three values are shown in figure 12(a) for the upper triangularity, the ' $\mathrm{X}$ ' represents the relevant point on the separatrix.

The ratio between the inner and outer scrape-off layer width is dependent on the 
triangularity, if vertical drifts determine the scrape-off layer width. The averaged parallel flow $v_{\|}$is assumed to be constant. Following the derivation in [21]:

$$
\lambda=\frac{\Delta \Psi_{p}}{\left|\nabla \Psi_{p}\right|}
$$

$\lambda$ being the scrape-off layer width, $\Delta \Psi_{p}$ is the displacement and $\left|\nabla \Psi_{p}\right|$ the gradient of the poloidal flux. The Maxwellian averaged grad B and curvature drifts are:

$$
\left\langle\boldsymbol{v}_{D}\right\rangle=\frac{2 T}{e Z R B} \hat{\boldsymbol{z}}
$$

with $T$ the temperature, $e$ the elementary charge, $Z$ the ion charge, $R$ the major radius, $B$ the magnetic field strength and $\hat{z}$ the unit vector in $z$ (vertical) direction. The displacement in the poloidal flux between the mid plane MP ( outer or inner) and the highest (lowest) point $S_{x}$ is:

$$
\Delta \Psi_{p}=\frac{1}{v_{\|}} \int_{M P}^{S_{x}}\left(\left\langle\boldsymbol{v}_{D}\right\rangle \cdot \nabla \Psi_{p}\right) d l_{\|}
$$

With the poloidal magetic field $B_{p}$

$$
B_{p}=\frac{\left|\nabla \Psi_{p}\right|}{R}
$$

we integrate in the poloidal plane along the flux surface

$$
\begin{aligned}
\Delta \Psi_{p} & =\frac{1}{v_{\|}} \int_{M P}^{S_{x}}\left(\left\langle\boldsymbol{v}_{D}\right\rangle \cdot \nabla \Psi_{p}\right) \frac{B}{B_{p}} d l_{p} \\
& =\frac{1}{v_{\|}} \int_{M P}^{S_{x}}\left(\left\langle\boldsymbol{v}_{D}\right\rangle \cdot \frac{\nabla \Psi_{p}}{\left|\nabla \Psi_{p}\right|}\right) R B d l_{p} \\
& =\frac{1}{v_{\|}} \int_{M P}^{S_{x}} \frac{2 T}{e Z R B} R B\left(\hat{\boldsymbol{z}} \cdot \frac{\nabla \Psi_{p}}{\left|\nabla \Psi_{p}\right|}\right) d l_{p} \\
& =\frac{2 T}{v_{\|} e Z} \int_{M P}^{S_{x}}\left(\hat{\boldsymbol{z}} \cdot \frac{\nabla \Psi_{p}}{\left|\nabla \Psi_{p}\right|}\right) d l_{p}
\end{aligned}
$$

for the last step we assumed a constant averaged $T$ and $Z$ along the integration path. Rewriting the direction of the poloidal flux gradient as

$$
\frac{\nabla \Psi_{p}}{\left|\nabla \Psi_{p}\right|} d l_{p}=\hat{\phi} \times d \boldsymbol{l}_{p}
$$

with $\hat{\phi}$ being the unit vector in toroidal direction. We write

$$
\begin{aligned}
\Delta \Psi_{p} & =\frac{2 T}{v_{\|} e Z} \int_{M P}^{S_{x}} \hat{\boldsymbol{z}} \cdot\left(\hat{\boldsymbol{\phi}} \times d \boldsymbol{l}_{\boldsymbol{p}}\right) \\
& =\frac{2 T}{v_{\|} e Z} \int_{M P}^{S_{x}}(\hat{\boldsymbol{z}} \times \hat{\boldsymbol{\phi}}) \cdot d \boldsymbol{l}_{\boldsymbol{p}}
\end{aligned}
$$

Using the previous definition of the triangularity $\delta$ the integral is solved as

$$
\int_{M P}^{S_{x}}(\hat{\boldsymbol{z}} \times \hat{\boldsymbol{\phi}}) \cdot d \boldsymbol{l}_{\boldsymbol{p}}=\int_{M P}^{S_{x}} \hat{\boldsymbol{R}} \cdot d \boldsymbol{l}_{\boldsymbol{p}}=a \cdot\left(1 \pm \delta_{x}\right)
$$


with '+' corresponding to the outer and '-' to the inner scrape-off layer width and $\hat{\boldsymbol{R}}$ the unit vector in radial direction. The displacement in the poloidal flux is hence

$$
\Delta \Psi_{p}=\frac{2 T}{v_{\|} e Z} a \cdot\left(1 \pm \delta_{x}\right)
$$

Therefore we can write the scrape-off layer width as

$$
\lambda=\frac{2 T}{v_{\|} e Z B_{p o l} R} a \cdot\left(1 \pm \delta_{x}\right)
$$

and the ratio between inner and outer scrape-off layer width is

$$
\frac{\lambda_{q}^{\text {inner }}}{\lambda_{q}^{\text {outer }}}=\frac{1-\delta_{x}}{1+\delta_{x}}
$$

both scrape-off layer widths are mapped to the pouter mid-plane.

For the case shown in figure 12(b) (USN and ion magnetic drift upwards) the upper triangularity $\delta_{\text {upper }}$ is determining the ratio between inner and outer scrape-off layer width.

$$
\left(\frac{\lambda_{q}^{\text {inner }}}{\lambda_{q}^{\text {outer }}}\right)_{\mathrm{B}_{\mathrm{t}}^{+}, \text {ion }}=\frac{1-\delta_{\text {upper }}}{1+\delta_{\text {upper }}}
$$

With a reversed drift direction, see figure 12(c) (USN and ion magnetic drift downwards), the lower triangularity $\delta_{\text {lower }}$ is determining the ratio between the inner and outer scrapeoff layer width.

$$
\left(\frac{\lambda_{q}^{\text {inner }}}{\lambda_{q}^{\text {outer }}}\right)_{\mathrm{B}_{\mathrm{t}}^{-}, \text {ion }}=\frac{1-\delta_{\text {lower }}}{1+\delta_{\text {lower }}}
$$

The interpretation of the r.h.s. of equations (22) and (23) are sketched in figure 12(b),(c) by the black two-headed arrows in the mid-plane. The left arrows represent $a(1-\delta)$, the right arrows $a(1+\delta)$.

Due to the opposite charge of electrons their respective drift directions are opposite to that of the ions. Therefore the determining triangularity is changing as well, with the upper triangularity for a negative toroidal magnetic field and lower triangularity for a positive toroidal magnetic field.

In figure 13 the ratio between inner and outer power fall-off length $\lambda_{q}$ is shown in dependence of the triangularity of the plasma for ions (a) and electrons (b) for both field directions.

The ratio between inner and outer power fall-off length $\lambda_{q}$ is described by the ion drifts whereas for electron drifts the ratio cannot be explained by the triangularity. Therefore it is concluded that in L-mode the existence of ion drifts can describe the observed asymmetry in the power fall-off length $\lambda_{q}$. The electron drifts cannot describe this asymmetry.

In this respect it is interesting to note that a comparison of the power fall-off length $\lambda_{q}$ in H-mode plasmas from JET with deuterium, hydrogen and helium as the main ion species with the heuristic drift based model by Goldston [21] was carried out. This work [11] came to the conclusion that the experimental estimated values for $\lambda_{q}$ did 


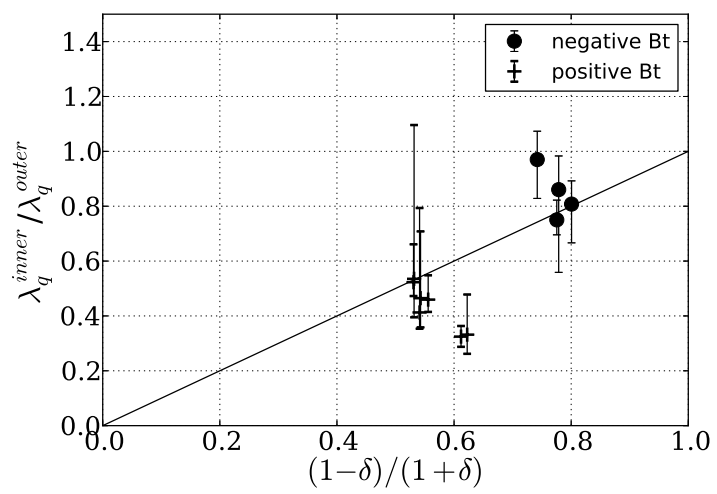

(a) ion drifts

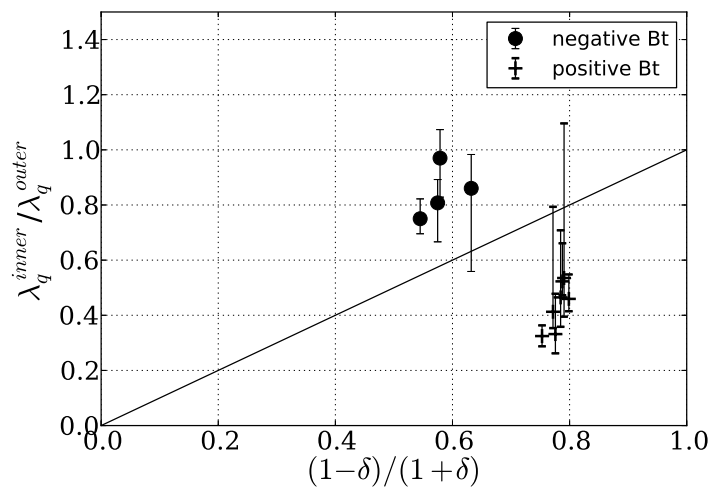

(b) electron drifts

Figure 13. Power fall-off length $\lambda_{q}$ : ratio between the value for the inner target divided by the value for the outer target versus $(1-\delta) /(1+\delta)$. For the left figure for positive toroidal magnetic field the upper triangularity $\delta_{\text {upper }}$ is taken, for negative toroidal magnetic field the lower triangularity $\delta_{\text {lower }}$. For the electrons the corresponding triangularities are used.

only agree to the Goldston-model when applying the isotope dependence as derived for the electron drift velocity. Our results here for L-mode plasmas in deuterium finds in contrast only agreement to the combined effect of vertical drifts and the triangularity for the ion drifts.

\subsection{Divertor broadening $S$}

In this section the divertor broadening $S$ measured in the open upper divertor of ASDEX Upgrade is compared to modelling results [23] as well as an empirical scaling law [24] for L-mode.

$$
S_{\text {scaling }}=0.09 \cdot n_{e, \text { edge }}^{1.02} \cdot B_{\text {pol }}^{-1.01}
$$

with the edge electron density $n_{e, \text { edge }}$ and the poloidal magnetic field $B_{\text {pol }}[24]$. The empirical scaling was derived from L-mode experiments that were conducted in ASDEX Upgrade LSN configuration with a closed divertor.

It is reported from modelling as well as experimental data that the divertor broadening $S$ is dependent on the target electron temperature [23]. Unfortunately there is no measurement for the target electron temperature $T_{e, \text { target }}$ in these discharges for the upper target.

The divertor broadening $S$ measured in USN does not show a strong dependence on the core electron density. The edge electron density measured by ThomsonScattering and the lithium beam [25] is closely coupled to the core electron density in these discharges as shown in figure 14(a). Figure 14(b) shows the measured divertor broadening $S$ against the empirical scaling law for LSN L-mode (equation (24)). 


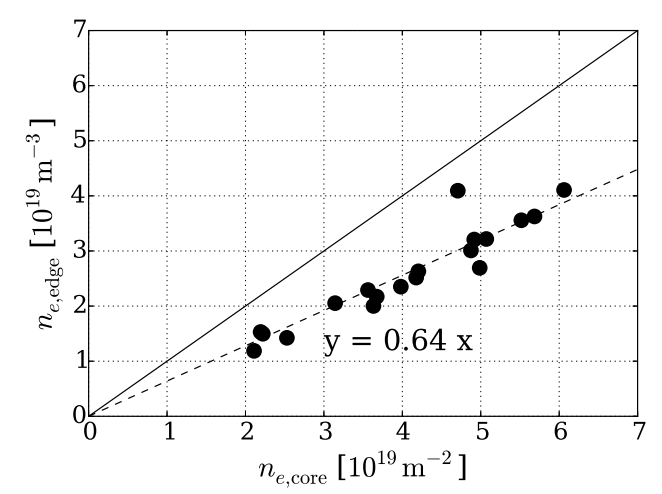

(a) Edge electron density in dependence of the core electron density.

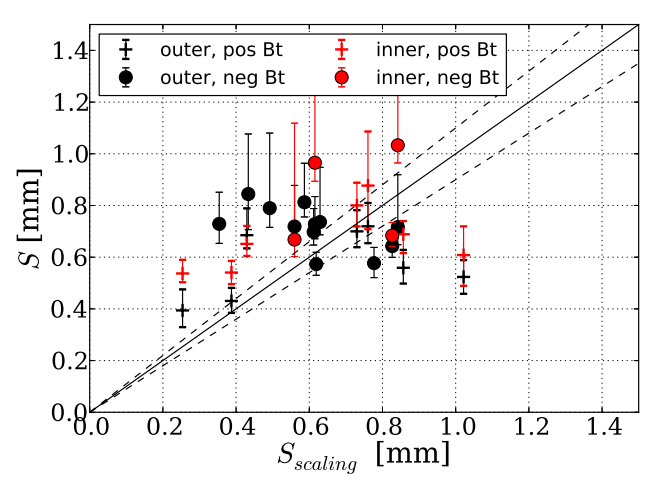

(b) Comparison between the divertor target divertor broadening $S$ and the values obtained using equation (24).

Figure 14. Edge electron density and scaling law for the divertor broadening $S$.

It is seen that the empirical scaling does not describe the data. The poloidal magnetic field is not varied within the data base and therefore the comparison to the scaling law is mainly the density dependence. A possible explanation is the different divertor closure for both configurations. Since for different divertor geometries different dependencies for the divertor electron density $n_{e, d i v}$ and target temperature $T_{e, \text { target }}$ are present the scaling for the closed divertor cannot be transferred to the open upper divertor. We attribute this to the observation that the divertor density in the open configuration is less coupled to the edge electron density compared to the closed divertor.

As mentioned above for the discharges investigated no target temperature $T_{e, \text { target }}$ measurements exist. A lower target electron temperature on the inner divertor could explain the larger divertor broadening $S$ values for the inner divertor target.

It is shown in [23] that the divertor broadening $S$ changes significantly only at target electron temperatures below $10 \mathrm{eV}$. One major concern for the discharges in this data base was that the divertor targets stay in attached conditions in order to be able to measure with the IR system. This might have led to high target electron temperatures. Therefore one possible explanation for the density independence could be a high target electron temperature.

However, we draw the conclusion from our studies here that for a generalized scaling of $S$ for different devices, despite the good description for the lower outer divertor with the scaling in equation (24), upstream parameters might not be a good choice.

\section{Summary}

L-mode experiments in USN were performed to study the scrape-off layer transport. The toroidal magnetic field direction is changed to investigate the effects of the magnetic drifts. For the divertor broadening $S$ no change with the reversed magnetic field is 
detected. The ratio between inner and outer power fall-off length $\lambda_{q}$ changes with the reversal of the toroidal magnetic field. This effect can be explained by the vertical magnetic drifts of ions and the triangularity of the plasma. Measurements with Thomson-Scattering and IR thermography are in agreement with the 2-point model for the outer divertor target.

For the inner divertor the 2-point model is not applicable between the outer midplane and the divertor target.

\section{Acknowledgements}

The authors are grateful for help from and discussions with A. Scarabosio and R. J. Goldston.

\section{Appendix}

Determination of the integral power decay width $\lambda_{\text {int }}$

Within measurement accuracy the comparison between the simple estimation of $\lambda_{\text {int }}$ from the model parameters, $\lambda_{q}$ and $S$, and the integration along the target is a good qualifier. However, as shown in figure 5 the comparison shows a deviation of around $3 \%$. This is because both ways of estimating the integral power decay length $\lambda_{\text {int }}$ are approximations with systematic errors.

Starting with the integration along the target (equation (1)). In an experimental environment the target that is observed is finite. This leads to an underestimation of $\lambda_{\text {int }}$. The observed divertor target is in the order of 4-5 times the power fall-off length $\lambda_{q}$. Therefore the exponential part of the profile is cut off after around 3 decay lengths. Taking an exponential decay only for simplicity:

$$
q(s)=q_{0} \exp \left(\frac{s}{\lambda_{q}}\right)
$$

The integration from $s=0$ to infinity:

$$
\int q(s) \mathrm{d} s=q_{0} \cdot \lambda_{q}
$$

and the given definition, $\lambda_{\text {int }}$ is equivalent with $\lambda_{q}$. With a finite upper limit of 3 times the decay width:

$$
\int_{0}^{3 \lambda_{q}} q(s) \mathrm{d} s=q_{0} \cdot \lambda_{q}(1-\exp (-3))
$$

the measured value of $\lambda_{\text {int }}$ is $5 \%$ lower than the real one. The measured value will always be smaller than the real value, but it cannot be quantified exact without assuming a model for the decay. The finite target for the Gaussian is negligible ( $\ll 1 \%$ error) if the observed profile in the private flux region is larger than $3 S$. This holds true for all discharges shown in this paper. 
The second part can be explained by the relation shown by Makowski [14]. The identity of equation (3) is only true for $S=0$. For finite $S$ values this equation is only an approximation. The error of this is dependent on the ratio between $S$ and $\lambda_{q}$. For values of $S / \lambda_{q}$ between 0.2 and 1 , as within the used data base, this formula overestimates the integral power decay width approximately by $2-4 \%$ [26].

In general the measured $\lambda_{\text {int }}$ from the integration along the target will always underestimate in the range of a few percent for a large enough observed target. The simple estimation taking the fitted values for $S$ and $\lambda_{q}$ will overestimate the integral power decay width $\lambda_{\text {int }}$. Whereas the error from the simple estimation can be calculated exactly, the finite observed target leads to an uncertainty with a lower limit for the real value for $\lambda_{\text {int }}$ with the measured value.

The regressed slope of 1.03, as mentioned in section 3.1, is, within the experimental uncertainties, in line with the expected systematic errors.

\section{References}

[1] LOARTE, A. et al., Nuclear Fusion 47 (2007) S203.

[2] WENNINGER, R. P. et al., Nuclear Fusion 54 (2014) 114003.

[3] ASDEX Team, Nuclear Fusion 291959.

[4] CARlstrom, T. N. et al., Plasma Physics and Controlled Fusion 40 (1998) 669.

[5] CORDEY, J. G. et al., Plasma Physics and Controlled Fusion 38 (1996) 1905.

[6] SNIPES, J. et al., Nuclear Fusion 36 (1996) 1217.

[7] PITTS, R. A. et al., Journal of Nuclear Materials 337339 (2005) 146.

[8] HERRMANN, A. et al., Plasma Physics and Controlled Fusion 37 (1995) 17.

[9] FUNDAMENSKI, W. et al., Journal of Nuclear Materials 337339 (2005) 305, PSI-16.

[10] STROTH, U. et al., Nuclear Fusion 53 (2013) 104003.

[11] EICH, T. et al., Phys. Rev. Lett. 107 (2011) 215001.

[12] MLYNEK, A. et al., Review of Scientific Instruments 81 (2010) 033507.

[13] SCHNEIDER, W. et al., Fusion Engineering and Design 48 (2000) 127 .

[14] MAKOWSKI, M. A. et al., Physics of Plasmas (1994-present) 19 (2012) 056122.

[15] SCARABOSIO, A. et al., Journal of Nuclear Materials 438, Supplement (2013) S426 .

[16] EICH, T. et al., Nuclear Fusion 53 (2013) 093031.

[17] KURZAN, B. and MURMANN, H. D., Review of Scientific Instruments 82 (2011) .

[18] NEUHAUSER, J. et al., Plasma Physics and Controlled Fusion 44 (2002) 855.

[19] STAnGEBY, P. C., The Plasma Boundary of Magnetic Fusion Devices, IoP, 2000.

[20] STANGEBY, P. C. et al., Nuclear Fusion 50 (2010) 125003.

[21] GOLDSTON, R. J., Nuclear Fusion 52 (2012) 013009.

[22] LUCE, T. C., Plasma Physics and Controlled Fusion 55 (2013) 095009.

[23] SCARABOSIO, A. et al., Journal of Nuclear Materials (2014) .

[24] SIEGLIN, B. et al., Plasma Physics and Controlled Fusion 55 (2013) 124039.

[25] WOLFRUM, E. et al., Review of Scientific Instruments 64 (1993) 2285.

[26] GOLDSTON, R. J., personal communication. 